Grthens, J.H., RosenkrantZ, J.G. \& TunNock, S.M. (1965) Teratogenic effects of azathiaprine (Muran). J. Pediat. 66, 959.

HARVAlD, B. (1967) European views on liver disease. Brit. med. J. 1, 232.

JACKSON, W.B. (1962) Lupoid hepatitis. N.Z. med. J. 61, 302.

MCArthur, J.W. \& FlaX, M.H. (1968) Amenorrhoea, hepatic disease and massive intra-abdominal haemorrhage. New Engl. J. Med. 278, 323

Mackay, I.R., Weiden, S. \& Ungar, B. (1964) Treatment of active chronic hepatitis and lupoid hepatitis with 6-mercaptopurine and azathiaprine. Lancet, i, 899.

Mistilis, S. \& BlackburN, C.R.B. (1967) Treatment of active chronic hepatitis with 6-mercaptopurine and azathiaprine. Aust. Ann. Med. 16, 305.
Moore, R.M. \& Hughes, P.K. (1960) Cirrhosis of the liver in pregnancy: a review of the literature and report of three cases. Obstet. Gynec. 15, 753.

Rosenkrantz, J.G., Githens, J.H., Cox, S.M. \& Kellum, D.L. (1967) Azathiaprine (Imuran) and pregnancy. Amer. J. Obstet. Gynec. 97, 387.

Seedat, Y.K. \& RaINe, E.R. (1965) Active chronic hepatitis associated with renal tubular acidosis and successful pregnancy. S. Afr. med. J. 39, 595.

Slater, R.J. (1954) Investigations of an infant born of a mother suffering from cirrhosis of the liver. J. Paediat. 13,308 .

\title{
Paroxysmal nocturnal haemoglobinuria with fatal puerperal stroke due to sagittal sinus thrombosis*
}

\author{
J. F. DAVIDSON \\ I. MacLean \\ M.B., M.R.C.P.(Ed.) \\ M.B., Ch.B., D.R.C.O.G. \\ Senior Registrar in Haematology \\ Royal Infirmary, Glasgow \\ Registrar in Obstetrics and Gynaecology \\ J. Hume Adams \\ M.B., Ph.D., M.C. Path. \\ Senior Lecturer in Neuropathology \\ D. I. GRaham \\ M.B., Ch.B. \\ Lecturer in Neuropathology \\ J. S. CARgill \\ M.B., Ch.B. \\ Registrar in Laboratory Medicine \\ Western Infirmary and Institute of Neurological Sciences, Glasgow
}

PAROXYsMal nocturnal haemoglobinuria (PNH) is an uncommon disorder with an approximate incidence of two per million population (Crosby, 1953). Pregnancy is a rather rare event in $\mathrm{PNH}$, and although until the time of delivery no especial risk has been reported, the puerperium is generally stormy. In a comprehensive review based on fifty-six cases of PNH, Dacie (1967) records three cases who became pregnant after its onset, one of whom developed thrombo-embolic disease.

In the present case, PNH was diagnosed at the time of delivery. Early in the puerperium a severe haemolytic episode developed which was followed by a slowly progressive and eventually fatal stroke

\footnotetext{
* This case has been presented to the Caledonian Branch of the Association of Clinical Pathologists and the British Neuropathological Society.
}

due to sagittal sinus thrombosis. Although venous thrombosis is common in PNH, sagittal sinus thrombosis is a rare event and its occurrence in a young post-partum woman with PNH has not previously been reported. Of particular interest in this case was evidence suggestive of reduced fibrinolytic activity during an episode of venous thrombosis.

\section{Case report}

A 34-year-old woman developed a transient right hemiparesis in October 1967 during the 28th week of her second pregnancy. An air encephalogram at that time showed an expanding lesion in the left cerebral hemisphere. She made a full spontaneous recovery over a period of 7 days. A macrocytic anaemia was noted and oral iron and folic acid were given for the rest of the pregnancy but without 
producing any significant rise in the haemoglobin level. The remainder of the pregnancy was uneventful until the 39th week when abdominal pain developed.

In the previous medical history it transpired that in 1960, the patient had developed a severe hypoplastic anaemia thought at that time to be secondary to the long acting sulphonamide sulphamethoxypyridazine (Lederkyn). The diagnosis was made on the basis of anaemia, thrombocytopenia and marrow hypoplasia and was supported by ferrokinetic studies. The haemoglobin level was maintained with blood transfusion and a full recovery was achieved in 6 months. Thereafter, she remained well and her first pregnancy in 1964 passed without obstetric or haematological incident.

On admission to the Royal Infirmary in the 39th week of her second pregnancy, the patient was a thin sallow woman with pale mucous membranes and a smooth liver edge palpable at the costal margin. There was no splenomegaly, lymphadenopathy or icterus. Apart from a residual right facial weakness full neurological examination was negative.

Investigations. $\mathrm{Hb} 9.0 \mathrm{~g} / 100 \mathrm{ml}, \mathrm{PCV} 29 \%$, WBC $5600 / \mathrm{mm}^{3}$, platelet count $130,000 / \mathrm{mm}^{3}$. The peripheral blood showed anisocytosis, poikilocytosis, macrocytosis, polychromasia and a reticulocyte count of $13 \%$ : many poikilocytes showed atypical 'rat tail' projections. The blood group was $\mathrm{O}$ Rhesus (D) negative and both direct and indirect antiglobulin (Coombs') tests were negative. Urine analysis revealed excess urinary urobilinogen and large amounts of haemosiderin in the centrifuged deposit.

These findings indicated chronic intravascular haemolysis and a diagnosis of PNH was made when two positive Ham's acid serum tests were obtained on successive days. The Ham's test was carried out according to Dacie \& Lewis (1963) using heat inactivated serum as a negative control and red cells from a known case of PNH as a positive control.

Two days after admission the patient went into labour and an uncomplicated vertex delivery followed. A 7-day course of stilboestrol was given to suppress lactation. The first post-partum day was uneventful but in the 2nd day lower abdominal pain developed which over a 24-hr period became generalized and was associated with vomiting. The abdomen became distended and a tender liver edge became palpable 3 in. below the costal margin. The haemoglobin was $6.6 \mathrm{~g} / 100 \mathrm{ml}$ and the platelet count $90,000 / \mathrm{mm}^{3}$. The patient became icteric, the total serum bilirubin was $2.5 \mathrm{mg} / 100 \mathrm{ml}$, with $1.3 \mathrm{mg} / 100 \mathrm{ml}$ direct-reacting bilirubin; serum alkaline phosphatase $21 \mathrm{KA}$ units $/ 100 \mathrm{ml}$, the SGOT $202 \mathrm{SF}$ units/100 $\mathrm{ml}$ and the SGPT $116 \mathrm{SF}$ units $/ 100 \mathrm{ml}$. The abdominal pain persisted and heavy continuous haemoglobinuria developed on the 5th post-partum day. The plasma haemoglobin level was $150 \mathrm{mg} / 100 \mathrm{ml}$ and the plasma methaemalbumin $3.0 \mathrm{mg} / 100 \mathrm{ml}$. This acute episode of intravascular haemolysis was managed symptomatically and blood transfusion was withheld. The haemoglobinuria disappeared by the 10th post-partum day, and the abdominal pain resolved 3 days later. Thereafter the patient's general condition improved, the haemoglobin rose to $7.9 \mathrm{~g} / 100 \mathrm{ml}$, the serum transaminases became normal and the liver edge could not be felt.

During the period of acute intravascular haemolysis, haemostatic and fibrinolytic investigations were carried out (Table 1). These were normal apart from an increased Factor $X$ level, a slightly increased Factor V level and a prolonged euglobulin lysis time. Serial serum samples at this stage were examined by

TABLE 1. Coagulation and fibrinolytic investigations carried out during intravascular haemolysis and hepatic vein thrombosis

\begin{tabular}{lcc}
\hline & Test & Normal/control \\
\hline Bleeding time (Duke Method, min) & 3 & $2-7$ \\
Whole blood clotting time (Lee and White, min) & 3 & $3-8$ \\
One stage prothrombin time (sec) & 15 & 15 \\
Kaolin cephalin clotting time (sec) & 40 & 35 \\
Thrombin time (sec) & 13 & 13 \\
Thromboplastin generation test & Normal & \\
Prothrombin consumption index (\%) & 22 & 40 \\
Factor V assay (\%) & 176 & $50-150$ \\
Factor VIII assay (\%) & 100 & $50-150$ \\
Factor IX assay (\%) & 100 & $50-150$ \\
Factor X assay (\%) & 450 & $50-150$ \\
Platelets/mm 3 & 200,000 & $150,000-400,000$ \\
Fibrinogen (mg/100 ml) & 267 & $200-400$ \\
Plasminogen (casein units/ml) & $3 \cdot 5$ & $2-4$ \\
Euglobulin lysis time (hr) & 11 & $2-10$ \\
\hline
\end{tabular}


an Ouchterlony technique using anti-human fibrinogen antiserum for fibrin degradation products but with negative results.

On the 24th post-partum day, the patient complained of headache, backache and abdominal pain and, $24 \mathrm{hr}$ later, had a grand mal seizure. These symptoms persisted and the liver edge was again palpable and tender. There was no haemoglobinuria.

On the 31st post-partum day a flaccid paresis of the left leg developed. There was no sensory loss, and the tendon reflexes and plantar responses were normal. Over the next 5 days headache became more severe and the paresis extended gradually to involve the left arm. Left facial pain developed.

As the haemoglobin level was again falling and the patient's condition deteriorating one unit of thrice washed red cells was given. Towards the end of the transfusion the headache intensified so further transfusion was withheld. The next day, 2 weeks after the onset of neurological symptoms, signs of acute brain stem compression appeared. The patient was transferred in coma to the Institute of Neurological Sciences where ventriculography showed small symmetrical ventricles and no shift of the midline structures. At the time of this procedure, anticipating a possible craniotomy, two units of thrice washed cells were transfused without any apparent reaction. In spite of further supportive measures, the patient died without regaining consciousness on the 40th post-partum day, 17 days after the onset of progressive neurological symptoms.

\section{Necropsy (K3/68)}

The anterior part of the superior sagittal sinus was occluded by old organized thrombus whereas the remainder contained more recent ante-mortem thrombus which had propagated throughout its length, into both sigmoid sinuses, and into the left jugular and subclavian veins.

The brain $(1350 \mathrm{~g})$ showed convolutional flattening and tentorial and tonsillar herniae. In coronal sections there was intensely haemorrhagic bilateral parasagittal infarction which was more extensive on the right (Fig. 1). Where infarction was severe, there was thrombosis of cortical veins but there was no arterial occlusion. In the left superior frontal gyrus there was an old partly yellow wedge-shaped infarct (Fig. 2). The ventricles were small and symmetrical and there were secondary haemorrhages in the pons. The cerebellum and spinal cord were normal.

In the heart $(300 \mathrm{~g})$ there was a $1.0 \mathrm{~cm}$ old fibrous scar in the posterior wall of the left ventricle. The aorta and its major branches, the venae cavae and the pulmonary trunk and arteries were patent but microscopy showed that some of the small pulmonary veins were occluded by thrombus. The capsular and cut surfaces of the enlarged liver $(2000 \mathrm{~g})$ had a

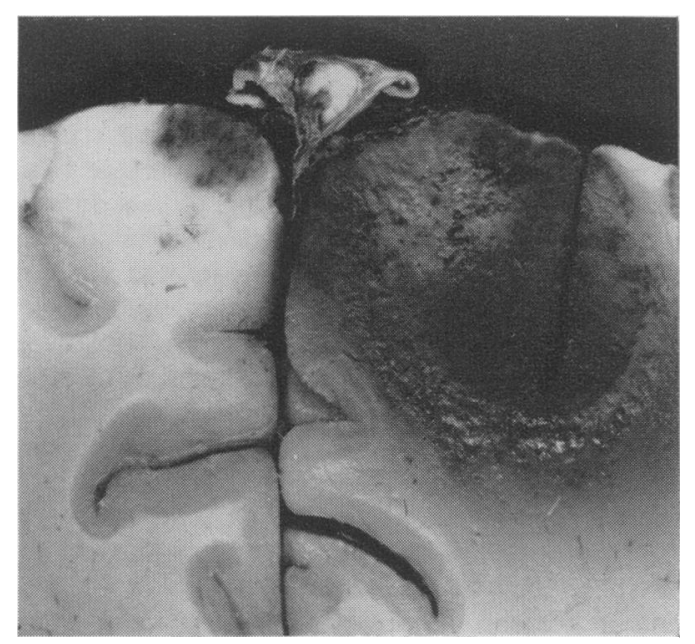

FIG. 1. Coronal section of fixed brain through parietal lobes. The superior sagittal sinus is occluded by thrombus and there is bilateral parasagittal venous infarction. This is much more extensive in the right hemisphere. $\times 1 \cdot 2$.

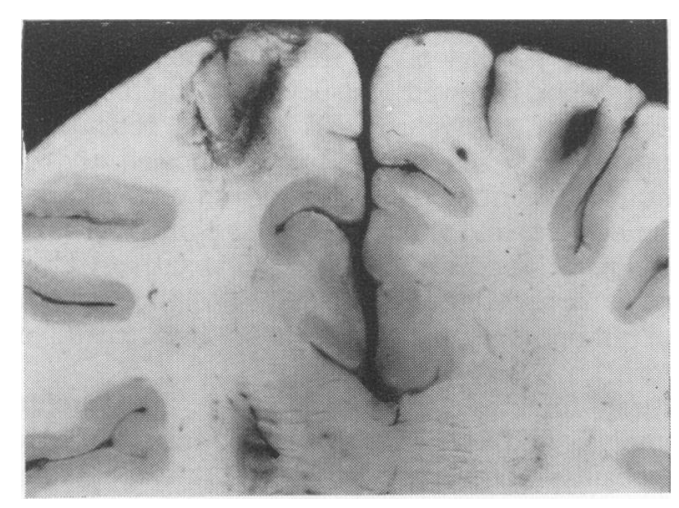

FIG. 2. Coronal section through frontal lobes. There is an old parasagittal venous infarct in the left superior frontal gyrus. $\times 0.8$.

coarsely mottled haemorrhagic appearance due to extensive recent centrilobular haemorrhagic and anaemic infarction. All of the major hepatic veins and many of their smaller branches were occluded by thrombus. The hepatic arterial and portal venous systems appeared normal although microscopy showed partial occlusion of an occasional small branch of the portal vein. The architecture of each kidney $(150 \mathrm{~g})$ was normal but the cortex had a distinct rust brown colour. The cut surface stained intensely blue with Perl's Prussian blue reaction and microscopy demonstrated heavy impregnation of the tubular epithelial cells with stainable iron. The spleen was enlarged $(250 \mathrm{~g})$ and the cut surface 
showed numerous recent infarcts due to occlusion of the splenic vein by thrombus. An infarct was also found in the anterior lobe of the pituitary, presumably due to raised intracranial pressure (Wolman, 1956).

There was no histological evidence of widespread thrombosis of small vessels in the many organs examined and stainable iron could be identified only in the kidneys.

\section{Discussion}

Hypoplastic anaemia, particularly the druginduced type, is known to be associated with PNH (Dacie, 1967). Marrow hypoplasia may precede the development of the disease or may complicate it. In this case marrow hypoplasia preceded overt PNH by 8 years. If a patient with chronic haemolytic anaemia gives a previous history of hypoplastic anaemia, PNH should be suspected, and Ham's acid serum test carried out (Dacie, 1967).

PNH is known to be associated with an increased thrombotic tendency (Scott, Robb-Smith \& Scowen, 1938; Manchester, 1945; Marks, 1949; Crosby, 1953; Dacie, 1967) but the mechanism of this is poorly understood and haemostatic investigations vary (Dacie, 1967). In this case the main haemostatic abnormality found was increased factor $\mathbf{X}$ activity (Table 1). The lack of fibrin degradation products in the serum plus the prolonged euglobulin lysis time at a stage when there was evidence of venous thrombosis is suggestive of a degree of inhibition of fibrinolytic activity. In the series of cases reviewed by Crosby (1953) approximately $50 \%$ died from cerebral and/or portal vein thrombosis. This patient illustrates the assocation well but the occurrence of thrombosis of the superior sagittal sinus in a patient with PNH appears to have been less commonly described although cases have been reported by Bergmark (1931), Ellenhorn et al. (1951) and Nussey \& Dawson (1956). Krayenbuhl (1967, personal communication 1968) in his series included one case of thrombosis of the anterior one-third of the superior sagittal sinus in a patient suffering from an aplastic-haemolytic anaemia of unknown origin.

In pregnancy and the puerperium there is an increased risk of thrombosis (Inman \& Vessey, 1968) and when stilboestrol is given to suppress lactation this risk is further increased (Daniel, Campbell \& Turnbull, 1967). This patient undoubtedly experienced episodes of superior sagittal sinus thrombosis in both pregnancy and the puerperium, the old venous infarct in the left superior frontal gyrus indicating the pathogenesis of the transient right hemiparesis experienced earlier in her second pregnancy. More recent antemortem thrombus in the posterior part of the superior sagittal sinus was clearly the cause of the terminal cerebral venous infarction. This patient's symptomatology and mode of presentation, therefore, correlated well with the autopsy findings.

It is commonly thought that the majority of strokes occurring in pregnancy and the puerperium are due to cerebral venous thrombosis. Although intracranial venous thrombosis does occur in pregnancy and more commonly in the puerperium (Kalbag \& Woolf, 1967), a recent survey undertaken in the West of Scotland by Cross, Castro \& Jennett (1968) shows that in a series of thirty-one patients who presented with strokes in pregnancy and the puerperium, and who were fully investigated, over $70 \%$ were due to occlusive cerebral arterial disease.

Blood transfusion, even with washed red cells, has been shown in some cases to aggravate haemolysis (Dacie, 1967). For this reason, transfusion was withheld and given only when the haematological state was deteriorating rapidly. In retrospect it might be said that the washed cell transfusion aggravated the haemolytic process.

Anti-coagulant therapy with coumarin preparations is not generally recommended in PNH (Dacie, 1967). Such treatment was considered in this case but rejected because of the ill defined nature of the abdominal pain and initial uncertainty as to the pathogenesis of the stroke.

\section{Acknowledgments}

Our thanks are due to Professor E. M. McGirr, Professor A.S. Douglas, Dr G. A. McDonald and Dr W. Black, of the Royal Infirmary, Glasgow and $\mathrm{Mr} \mathrm{A}$. Paterson of the Institute of Neurological Sciences, Glasgow.

\section{References}

Bergmark, G. (1931) Ein Fall von intermittierender kryptogener Hamogloginurie. Acta med. scand. 77, 224.

Crosby, W.H. (1953) Paroxysmal nocturnal haemoglobinuria: relation of the clinical manifestations to underlying pathogenic mechanisms. Blood, 8, 769.

Cross, J.N., Castro, P.O. \& JennetT, W.B. (1968) Cerebral strokes associated with pregnancy and the puerperium. Brit. med. J. 3, 214.

DACIE, J.V. (1967) The Haemolytic Anaemias, 2nd edn, p. 1128. Churchill, London.

DaCie, J.V. \& Lewis, S.M. (1963) Practical Haematology, 3rd edn, p. 147. Churchill, London.

Daniel, D.G., Campbell, H. \& Turnbull, A.C. (1967) Puerperal thromboembolism and suppression of lactation. Lancet ii, 287.

Ellenhorn, M.J., Feigenbaum, L.Z., Plumhof, C. \& Mettier, S.R. (1951) Paroxysmal nocturnal hemoglobinuria with chronic hemolytic anaemia. Arch. intern. Med. $87,868$.

INMAN, W.H.W. \& VESSEY, M.P. (1968) Investigation of deaths from pulmonary coronary and cerebral thrombosis and embolism in women of child bearing age. Brit. med. J. 2, 193.

KalbaG, R.M. \& Woolf, A.L. (1967) Cerebral Venous Thrombosis, pp. 148 and 155. Oxford University Press, London.

Krayenduht, H.A. (1967) Clinical Neurosurgery. Proceedings of the Congress of Neurological Surgeons, Vol. 14, p. 2. Williams \& Wilkins, Baltimore. 
MANChester, R.C. (1945) Chronic haemolytic anaemia with paroxysmal nocturnal haemoglobinuria. Ann. intern. Med. 23, 935.

MARKS, J. (1949) The Marchiafava Micheli syndrome. Quart. J. Med. 18, 105.

Nussey, A.M. \& Dawson, D.W. (1956) Paroxysmal nocturnal haemoglobinuria. Case study, including evidence of affection of the marrow in the disease. Blood, 11, 757.

ScotT, R.B., Roвb-Smith, A.H.T. \& Scowen, E.F. (1938) The Marchiafava-Micheli syndrome of nocturnal haemoglobinuria with haemolytic anaemia. Quart. J. Med. 8, 95 .

Wolman, L. (1956) Pituitary necrosis in raised intracrania pressure. J. Path. Bact. 72, 575.

\section{Adult hypertrophic pyloric stenosis}

\author{
N. A. M. SAlmo \\ Ph.D.(Manchester) \\ Department of Pathology \\ Faculty of Medicine, University of Mosul, Mosul, Iraq
}

HYPERTROPHY of the muscle fibres of the pylorus in the adult is a curious lesion that deserves more frequent consideration, especially in the differential diagnosis of constricting lesions of the distal stomach. Although male predominance is not as marked in adult as in infantile hypertrophic pyloric stenosis, men exceed women in the ratio of $3: 1$ (Andresen, Gammelgaard \& Licht, 1946; North \& Johnson, 1950). The great majority of adults presenting with symptoms referable to pyloric muscle hypertrophy are between 30 and 60 years of age. The age range, however, is wide; some symptomatic cases have been observed in patients in the ninth decade of life (Albot \& Magnier, 1955).

The present report describes a case of hypertrophic pyloric stenosis in a young female, adding one to the small number recorded.

\section{Case report}

An unmarried female aged 15 years was admitted to the Republican Hospital on 7 September 1966 complaining of vomiting of 6 months' duration. The vomiting was at first mild and sparse, later it became frequent and copious. It was unrelated to the type of food. The vomitus was invariably sour in taste and contained undigested food particles. There was no history of haematemesis or melaena. There had been some weight loss.

On admission she appeared slightly wasted but not acutely ill. No abnormalities were found in the heart, lungs, abdomen, urinary system and the central nervous system. The liver and spleen were not palpable. Her temperature was $36.8^{\circ} \mathrm{C}$, the blood pressure was $110 / 70 \mathrm{mmHg}$ and the pulse was $90 / \mathrm{min}$.
Investigations. $\mathrm{Hb} 11.8 \mathrm{~g} / 100 \mathrm{ml}$, ESR $53 \mathrm{~mm} / \mathrm{hr}$ (Westergren); WBC $7650 / \mathrm{mm}^{3}$ with a normal differential count. The stool contained no occult blood. Chest radiograph was normal.

The patient received chlorpromazine, ferrous sulphate and vitamin B complex daily for 12 days with no benefit. On barium meal examination (Fig. 1) the stomach was found dilated and ptosed; the duodenal cap was not visualized in all the projections; no barium distal to the gastric antrum could be detected; hypertrophy of the gastric mucosal pattern was noted. The findings were in favour of a long-standing pyloric obstruction. In view of this, the patient was transferred to the surgical side on 20 September 1966 for operation. Pre-operatively she received iron intramuscularly, vitamin B complex and vitamin $C$. The operation was performed on 9 October 1966.

Operation. The stomach was exposed through an upper median incision extending down to the umbilicus. It was found to be greatly dilated and there was no evidence of ulceration. The viscera were normal. A soft mass was felt in the pyloric region and a small incision was made through it. A villouslike obstruction was found in the pyloric canal, the lumen of which measured no more than a few millimeters in diameter. A localized partial gastrectomy with gastro-duodenostomy was performed. The regional sub-pyloric lymph nodes were removed and were sent with the excised pylorus for histopathological examination.

The post-operative course was uneventful. At follow-up, the patient showed a moderate gain in weight, good appetite and no dyspeptic symptoms. A post-operative barium meal showed a normal- 